\title{
Two-session endoscopic purse-string suture to close a huge esophagojejunal anastomosis thoracic cavity fistula
}

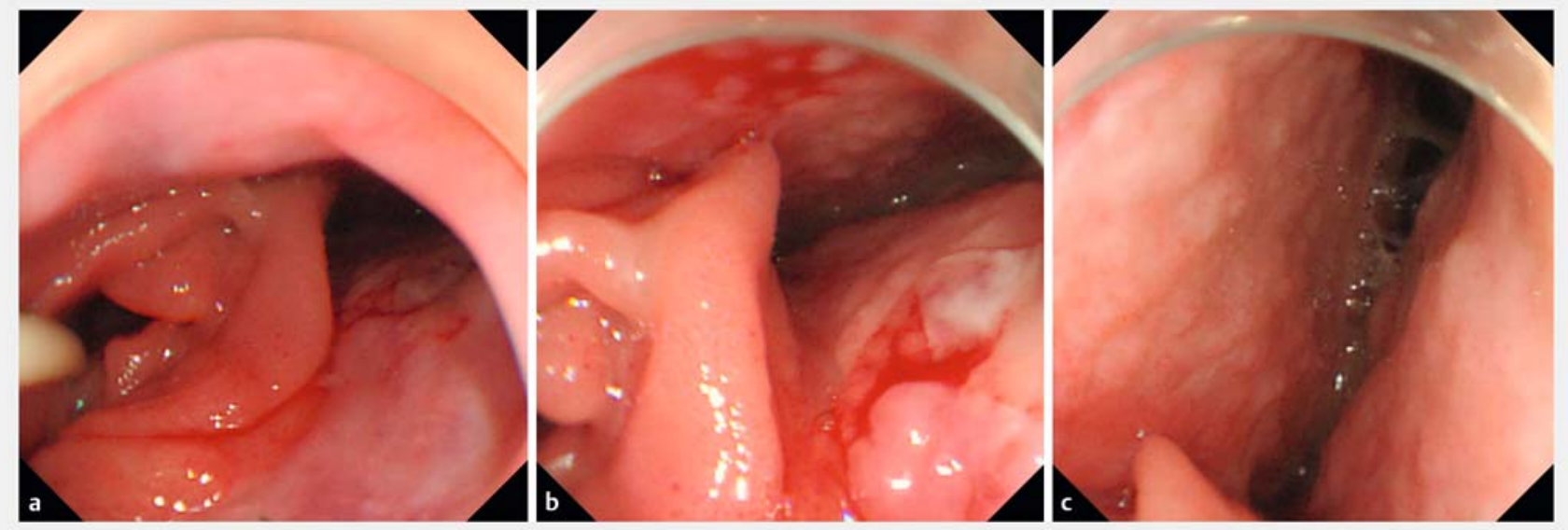

- Fig. 1 A huge esophagojejunal anastomosis thoracic cavity fistula occurred after laparoscopic distal esophagectomy and total gastrectomy. a The fistula located at the right wall of the anastomosis. $\mathbf{b}$ The mucosa of the fistula was soft and smooth. $\mathbf{c}$ No milky pus or sphacelus had collected in the thoracic cavity.

Esophageal anastomosis leaks or fistulas are life-threatening conditions after surgery and have high morbidity and mortality. Traditionally, neither surgical repair nor conservative therapy could improve the prognosis effectively. In recent years, endoscopic methods, such as using esophageal stents, OverStitch (Apollo Endosurgery, Austin, Texas, USA), and the Over-the-Scope Clip system (Ovesco Endoscopy AG, Tübingen, Germany), have been an effective alternative to surgery [1-3]. However, it remains a challenge to endoscopically close huge fistulas, especially those larger than $30 \mathrm{~mm}$. We report the method of endoscopic purse-string suture with clips and endoloops for the closure of a huge esophagojejunal anastomosis fistula [4].

A 74-year-old man diagnosed with cardiac carcinoma underwent laparoscopic distal esophagectomy and total gastrectomy. However, he presented with progressive shortness of breath 5 days after surgery. Upper gastrointestinal imaging showed anastomosis leakage. Gastroscopy revealed a huge esophagojejunal anastomosis thoracic cavity fistula of approxi-
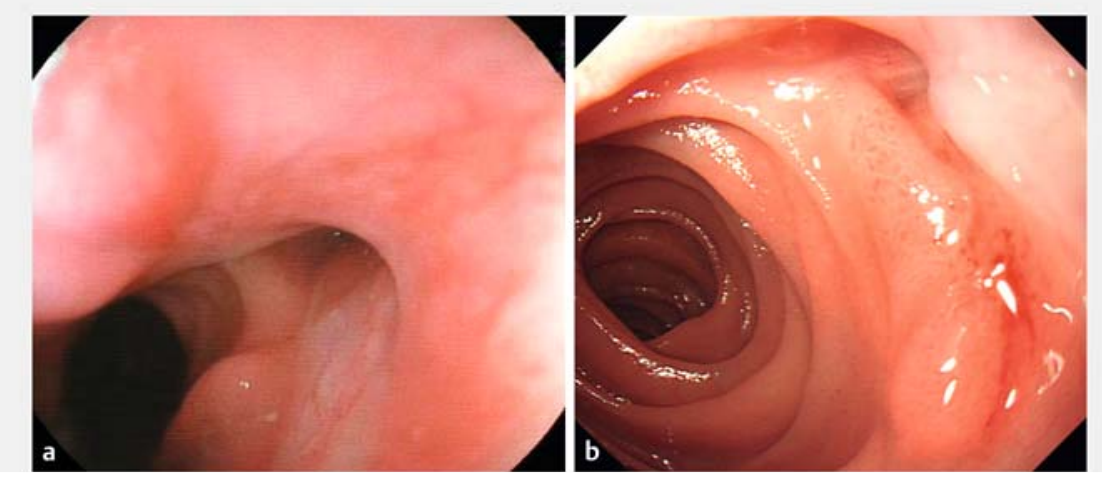

- Fig. 2 Closure of the fistula by endoscopic purse-string suture. a The fistula significantly decreased in size 1 month after the first procedure. b The fistula had completely closed 1 month after the second procedure.

mately $35 \mathrm{~mm}$ in size located at the right wall of the anastomosis ( $>$ Fig. 1 ). A nasojujunal feeding tube was implanted deeply beyond the fistula. Gastroscopy 2 weeks later revealed that the fistula had not significantly decreased. Given the poor condition of the patient, surgery was abandoned and closure of the fistula by endoscopic purse-string suture was attempted ( $\triangleright$ Video 1 ).
A 3-cm loop (MAJ-254; Olympus, Tokyo, Japan) was selected according to the fistula size. After adjustment of the location and angle of the endoloop, it was anchored onto the edge of the fistula with nine clips at different sides, aided by simultaneously tucking the edge with slight suction. The defect was then successfully closed by tightening the loop. Gastroscopy 1 month later revealed 


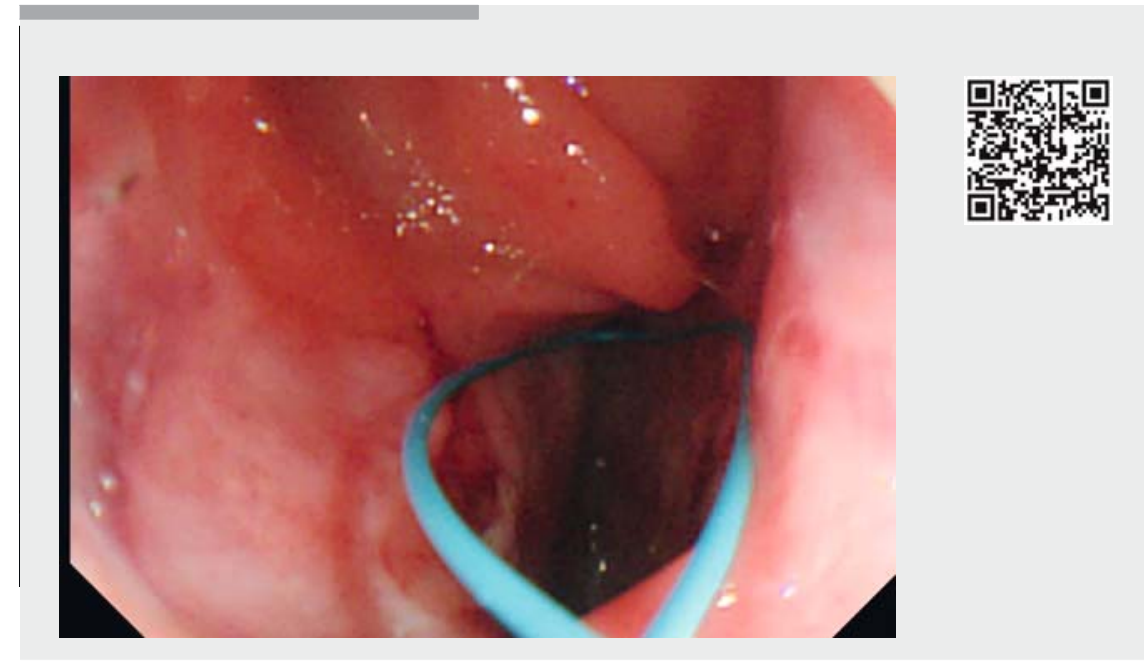

$\checkmark$ Video 1 Two-session endoscopic purse-string suture to close a huge esophagojejunal anastomosis thoracic cavity fistula in a 74-year-old man.

that the fistula had decreased to $10 \mathrm{~mm}$ in size; endoscopic purse-string suture was repeated ( $\mathbf{F i g} \mathbf{2} \mathbf{a})$. Gastroscopy 1 month after this repeat procedure showed that the defect had completely closed ( $\triangleright$ Fig. 2 b).

This case suggests that endoscopic purse-string suture may be an effective and feasible method for closure of huge anastomosis fistulas, but multiple sessions might be needed.

Endoscopy_UCTN_Code_TTT_1AO_2AC

\section{Competing interests}

None

\section{References}

[1] Oprisanescu D, Bucur D, Sandru V et al. Endoscopic treatment of benign esophageal fistulas using fully-covered metallic esophageal stents. Chirurgia (Bucur) 2018; 113: $108-115$

[2] Chon SH, Toex U, Plum PS et al. Successful closure of a gastropulmonary fistula after esophagectomy using the Apollo Overstitch and endoscopic vacuum therapy. Endoscopy 2018; 50: E149-E150

[3] Lee HL, Cho JY, Cho JH et al. Efficacy of the over-the-scope clip system for treatment of gastrointestinal fistulas, leaks, and perforations: A Korean multi-center study. Clin Endosc 2018; 51: 61-65

[4] Shi Q, Chen T, Zhong YS et al. Complete closure of large gastric defects after endoscopic full-thickness resection, using endoloop and metallic clip interrupted suture. Endoscopy 2013; 45: 329-334

\section{Bibliography}

DOI https://doi.org/10.1055/a-0658-0995

Published online: 22.10.2018

Endoscopy 2019; 51: E1-E2

(c) Georg Thieme Verlag KG

Stuttgart · New York

ISSN 0013-726X Hospital Affiliated to the Second Military Medical University, Shanghai, PR China

2 Department of Gastrointestinal Surgery, Changhai Hospital Affiliated to the Second Military Medical University, Shanghai, PR China

Corresponding author

\section{Zhendong Jin, MD}

Department of Gastroenterology, Changhai Hospital Affiliated to the Second Military Medical University, Building No. 11, Changhai Road No.168, Yangpu District, Shanghai 200433, PR China Fax: +86-21-31161336 jinzhendong2017@163.com 